\title{
Prospects of Physics at CDF with the SVX
}

\author{
The CDF Collaboration \\ Fermi National Accelerator Laboratory \\ P.O. Box 500, Batavia, Illinois 60510 \\ Presented by S. Dell'Agnello
}

September 1991

* Presented at the 3rd Topical Seminar on Heavy Flavors, S. Miniato, Italy, June 1991. 


\title{
Prospects of Physics at CDF with the SVX
}

\author{
Presented by S. Dell'Agnello (INFN-Pisa) for the CDF Collaboration \\ at the '3rd Topical Seminar on Heavy Flavors', S. Miniato, June 1991, Italy
}

\begin{abstract}
During next physics run CDF will strongly enhance its heavy flavor tagging capabilities with the installation of a silicon vertex detector (SVX), that will allow precise measurements of secondary decay vertices in the plane transverse to the beam (impact parameter resolution $\simeq 12 \mu \mathrm{m}$ ). We expect this detector to have a significant impact on b-physics $\left(c \tau_{B} \simeq 350 \mu \mathrm{m}\right)$ and top search. In the following we will discuss CDF prospects for top search and for CP violation asymmetry measurements in the B-sector.
\end{abstract}

\section{Physics Motivations for Vertex Detection}

CDF is in a privileged position to perform top searches [1] and study b-physics [2]. In fact, the TEVATRON provides $p \bar{p}$ collisions at the highest $\sqrt{s}$ currently available and this results into high cross sections both for top and beauty production. Several CDF upgrades are being commissioned to increase the efficiency for tagging heavy flavors; primarily the extension of the muon detector coverage and the installation of a silicon vertex detector.

With an integrated luminosity of $25 \mathrm{pb}^{-1}$ (the goal for the 1992 run), approximately $5 \times 10^{8}$ b's will be produced. At CDF the total inelastic cross section, $\sigma_{\text {inel }}$, is of the order of $50 \mathrm{mb}$ and the b-production cross section is $\sigma_{b} \simeq 20 \mu \mathrm{b}$ for $\left|y_{b}\right| \leq 1.0$ [3], which gives a ratio $\sigma_{b} / \sigma_{\text {inel }} \simeq 4 \times 10^{-4}$. The secondary vertex detection provided by the SVX $([4,5])$, in association with the good CDF lepton identification, will be essential for the required physical background rejection. We estimate that this can be done by preserving a good signal detection efficiency for B meson tagging. The SVX will allow detecting $\mathrm{B}$-decay modes (leptonic and non-leptonic) with an impact parameter (D) resolution $\sigma_{\mathrm{D}} \simeq 12 \mu \mathrm{m}$, that is fully adequate for B-tagging, given typical $D$ values of $\mathrm{B}$ sons $\left(\mathrm{c} \tau_{B} \simeq 350 \mu \mathrm{m}\right)$. This will be extremely useful in particular in the search for top, since top quark events contain always two b's (section 3 ), and for CP violation studies, where a neutral $B$ is selected to decay in an exclusive mode (section 4).

\section{SVX Performance}

The SVX (fig. 1,2) is built to provide high resolution $r-\phi$ tracking in a pseudorapidity interval $|\eta|<1.9$.

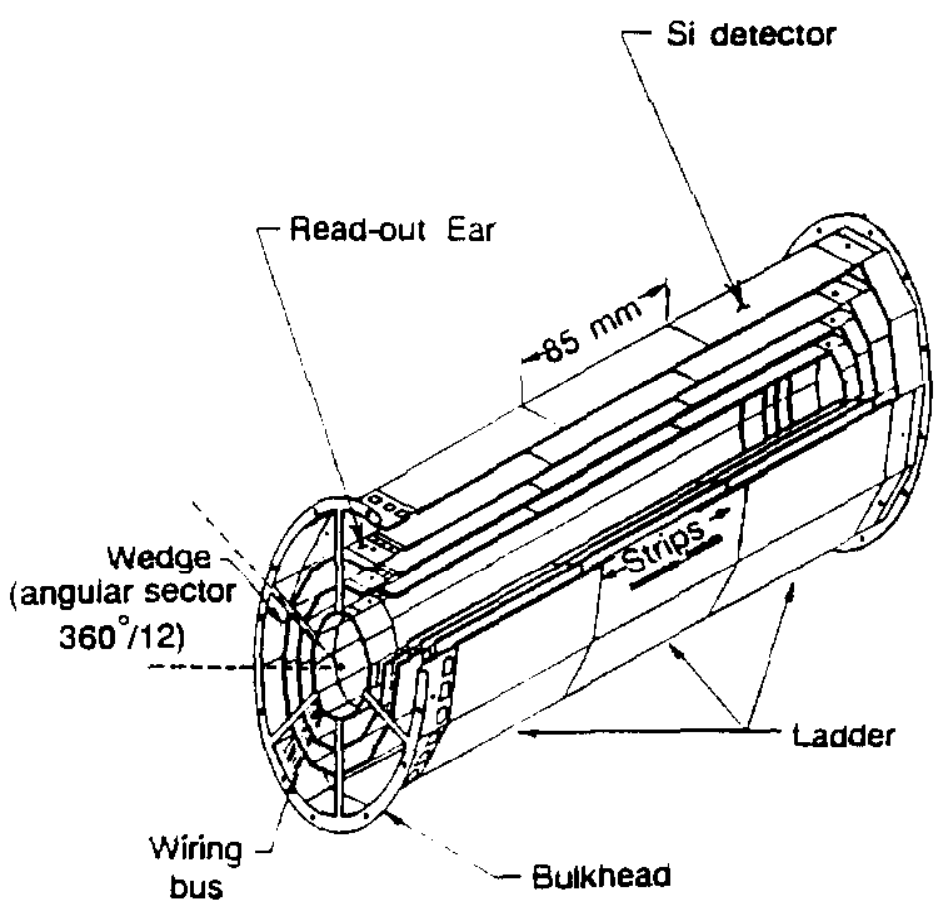

Figure 1: Isomeric view of one of the two SVX barrels 
Tagging the b's with the SVX will be essential, especially for the lepton + jets decays, because of the low rate of dileptons. CDF already tried to tag b's in the lepton + jets sample, by searching for low $P_{t}$ muons from sequential decays. In spite of its low efficiency, the low $-P_{t} \mu$ search, combined with results from the other modes, provided an increase of a couple of $\mathrm{GeV}$ in the lower limit on $M_{\text {top }}\{1\}$. By using the SVX for tagging b's we expect to get a larger improvement.

\section{$3.1 \quad$ B-tag Efficiency in Top Events}

Preliminary studies on B meson tagging efficiency in top decays have been performed using the ISAJET Montecarlo program as event generator and a simplified SVX detector simulation $[10,11]$. We assume the $\sigma_{D}$ curve of fig. 4 and we say that a $B$ has been tagged when there are at least 3 charged prongs within SVX +CTC acceptance and with $|D|>3 \sigma_{D}$. Table 1 shows the efficiencies for tagging at least 1 B (B1) and 2 B's (B2) in the event for three values of the top mass. The event z-vertex smearing gives $\simeq 60 \%$ contribution to $\mathrm{B} 1$ and B2.

Table 1: Efficiencies as a function of $M_{\text {top }}$

\begin{tabular}{|c|c|c|}
\hline $\begin{array}{c}M_{\text {top }} \\
\mathrm{GeV} / \mathrm{c}^{2}\end{array}$ & $\begin{array}{c}\mathrm{B} 1 \\
\%\end{array}$ & $\begin{array}{c}\mathrm{B} 2 \\
\%\end{array}$ \\
\hline 100 & 19 & 2 \\
150 & 28 & 4 \\
200 & 30 & 5 \\
\hline
\end{tabular}

More detailed studies are in progress in which we select a sample of $t \bar{t}$ events passing the cuts of the lepton + jets analysis described in $[1,8,9]$ and exploit correlations between impact parameter, $D$, and azimuthal production angle, $\phi$, of tracks coming from a secondary vertex (for a description of $D-\phi$ correlations see [12]). Preliminary results indicate that efficiencies increase with this method.

\section{$3.2 \mathrm{~S} / \mathrm{N}$ Improvement with the B-tag}

The physical background for the lepton + jets mode is given by direct $b \bar{b}$ production, gluon splitting $(g \rightarrow b \bar{b})$, $\gamma$ conversions and production of $\mathrm{W}$ bosons associated to QCD jets. To get rid of the background we require a high $P_{t}$ isolated lepton, large missing transverse energy $\left(E_{t}\right)$ and we cut on the associated jet Et's. After this selection $W+$ jets is the only non-negligible background left. This is no surprise, since the top decay is

$$
t \bar{t} \rightarrow W^{+} b W^{-} \bar{b}
$$

followed by

$$
W \rightarrow l+E_{t}, \quad W \rightarrow j e t j e t,
$$

which is to be compared with

$$
W+j e t s \rightarrow l+\mathrm{E}_{\mathfrak{t}}+j e t s,
$$

which basically leads to the same topology. However, a clear difference is given by the presence of the two b's in $t \bar{t}$ events. On the basis of present top analysis results, we think to be able to achieve a $\mathrm{S} / \mathrm{N}$ slightly better than 1 for $M_{\text {top }}=150 \mathrm{GeV} / \mathrm{c}^{2}$, without SVX information.

The upper limit to the $S / N$ improvement achievable with the $B$-tag is set by the $b$-content in $W+$ jets events. A conservative estimate of the upper limit can be done assuming that: a $) \simeq 3 \%$ of gluon jets $\left(P_{t}>10 \mathrm{GeV}\right)$ produce $b$ pairs $[13], b)$ there are in average 4 gluons in

$W+$ jets events, that is $\simeq 12 \%$ of $W+$ jets events have $b$ pairs; c) the percentage of tagging errors (fake secondary vertices) is negligible compared to the fraction of real b's in W+jets events (confirmed by preliminary studies); d) the tagging efficiency for b's from gluons is the same as for b's from top decays. With these assumptions the upper limit to the $\mathrm{S} / \mathrm{N}$ improvement is $100 / 12 \simeq 8$. In 
practice, we expect the efficiency for tagging b's from gluons to be lower because they have a lower $P_{t}$ than in top decays and therefore the improvement to be bigger. In this estimate we neglected other sources of physical background (like $c \bar{c}$ ), because we think that they are not large compared to the b-content in $\mathrm{W}+$ jets events; with next run's data we will have a good sample to measure them quantitatively. For example, the efficiency for tagging $D$ mesons should be significantly lower than for $B$ mesons, because of the lower $D$ lifetime and because of the lower $P_{\mathrm{t}}$ and multiplicity of $\mathrm{D}$ decays.

\section{CP Violation in the B-sector}

CDF has an extensive b-physics program, which may ultimately allow attacking the most ambitious goal, i.e. observing $\mathrm{CP}$ violation in the $\mathrm{B}$-sector. We are presently evaluating our chances, by studying the asymmetry in the decay rates of $B^{\circ}$ and $\bar{B}^{\circ}$ to the CP eigenstate $J / \Psi$ K.:

$$
A=\frac{\Gamma\left(B^{\circ} \rightarrow J / \Psi K_{s}\right)-\Gamma\left(\vec{B}^{\circ} \rightarrow J / \Psi K_{s}\right)}{\text { sum }} .
$$

The final state can be easily tagged by reconstructing $J / \Psi \rightarrow \mu^{+} \mu^{-}$and $K_{a} \rightarrow \pi^{+} \pi^{-}$. The flavor of the neutral $\mathrm{B}$ meson decaying to the $\mathrm{CP}$ eigenstate can obtained by looking at the charge of the lepton coming from the semileptonic decay of the other b-hadron.

An important parameter in this study is the integrated luminosity, $L$, that is needed in order to see CP violation with the CDF detector. The measured CP violating asymmetry, $A$, is proportional to $\sin 2 \beta$, the raw $\mathrm{CP}$ violating asymmetry from the CKM matrix $[14,15]$. The error on $\sin 2 \beta$ can be expressed as

$$
\delta(\sin 2 \beta)=\left(L d_{\text {phys }} d_{\text {ree }} d_{t a g}\right)^{-\frac{1}{2}}
$$

where $d_{\text {phys }}$ depends only on the physics process, $d_{\text {ree }}$ is a measure of how well $J / \Psi K$, events are reconstructed

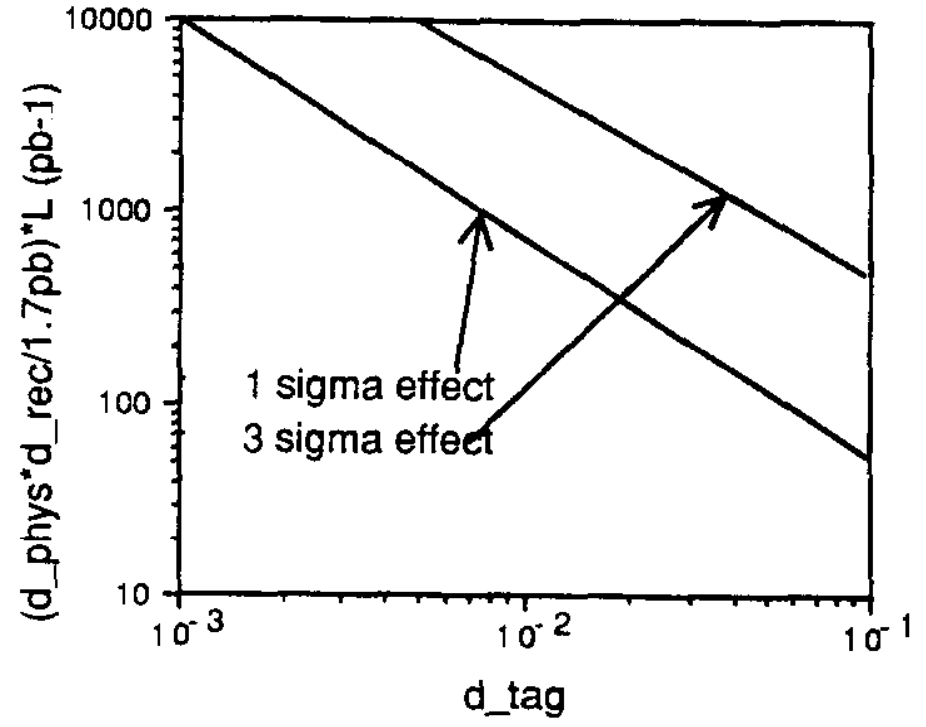

Figure 5: $L$ required to observe CP asymmetries

and $d_{t a g}$ is a measure of how well the flavor of the other b-hadron is tagged. Theoretical predictions [16] give $\sin 2 \beta=.34$ with an uncertainty $0.1<\sin 2 \beta<1.0$.

Extrapolating from present data and assuming significant detector upgrades for future runs, we estimate [15] that CDF could reach $d_{\text {phys }} \times d_{\text {ee }} \simeq 1.7 p \mathrm{~b}$ and $d_{\text {tag }} \simeq$ 0.01 (or $0.005<d_{\text {tag }}<0.05$ ). Fig. 5 shows that in order to measure the raw $\mathrm{CP}$ violation with an error $\delta(\sin 2 \beta) \leq \sin 2 \beta$ we need $L>500 \mathrm{pb}^{-1}$.

This integrated luminosity is not out of CDF's reach. if Fermilab accelerator will be upgraded with the construction of the new Main Injector (1995 or after). As far as the detector is concerned, in order to measure CP violation in the $J / \Psi K$, channel it is essential to have high reconstruction/tagging efficiency and good background rejection. The figures used in the previous estimates should be granted by the secondary vertex detection that will be provided by the SVX.

\section{Conclusions}

CDF expects a large physics outcome from 1992 run. In particular the enhanced tracking resolution provided by 
the SVX will increase our chances to discover the top quark. A measurement of CP violation in the B-sector may not be out of reach in the long term future, but requires an integrated luminosity in excess of $500 \mathrm{pb}^{-1}$.

\section{References}

[1] 'Heavy Flavor Physics from Colliders (UA1, UA2, CDF)', L. Galtieri for the CDF Collaboration, these proc.

[2] 'b-Physics at CDF and Prospects for the Next Run', H. Wenzel for the CDF Collaboration, these proc.

[3] P. Nason, S. Dawson, R.K. Ellis, Nuclear Physics B327 (1989), 49-92.

[4] 'The CDF SVX: a Silicon Vertex Detector for a Hadron Collider', D. Amidei et al, NIM A289 (1990) 388.

[5] 'Progress in the Construction of the CDF Silicon Vertex Detector', D. Amidei et al, presented by J. Skarha at the ' 5 th Pisa Meeting on Advanced Detectors', May 1991, Italy, Submitted to NIM.

[6] 'SVX Test Beam Results', D. Amidei et al., presented by S. Dell'Agnello at the '5th Pisa Meeting on Advanced Detectors', May 1991, Italy, Submitted to NIM.

[7] 'Search for New Heavy Quarks in Electron-Muon Event at the Fermilab Tevatron Collider', (CDF Collaboration), Phys. Rev. Lett. 64, 147 (1990).

[8] 'Search for the Top Quark in the Reaction $p \bar{p} \rightarrow$ Electron + Jets at $\sqrt{s}=1.8 \mathrm{TeV}$, (CDF Collaboration), Phys. Rev. Lett. 64, 142 (1990).

[9] 'Top-quark Search in the Electron + Jets Channel in Proton-Antipron Collisions at $\sqrt{s} 1.8 \mathrm{TeV}$, (CDF Collaboration), Phys. Rev. D 43, 664 (1991).

[10] 'Silicon Vertex Simulation, Tracking, Physics Estimates', F. Bedeschi et al, CDF internal note \# 601
[11] FNAL proposal P-775: to upgrade CDF with a silicon vertex detector in order to tag long-lived heavy flavors.

[12] 'Neural Networks for Triggering', F. Bedeschi et al, Preprint Fermilab - Conf-90/20, Submitted to IEEE Trans. on Nuclear Science.

[13] A.H. Mueller, P. Nason, Phys. Lett. 157B, 226 (1985)

[14] N. Mistry, T. Browder, CLEO internal note CBX90-43; CLNS 91-1043

[15] 'b Physics at CDF', edited by P. Tipton, CDF internal note \# 1482

[16] C.S. Kim, J.L. Rosner, C.P. Yuan, Phys. Rev. I

\section{$42,96(1990)$}

